\section{HEAD-DROPPING TEST}

BY

\section{ROBERT WARTENBERG, M.D.}

(From the University of California School of Medicine, San Francisco)

This test serves, first of all, in the early diagnosis of paralysis agitans and post-encephalitic Parkinsonism. The latter term is an awkward one, and has already led to such a stylistic horror as " Rombergism," to which "Babinskiism," it is hoped, will not be added. Therefore the generic term "Parkinson's syndrome" is used here, and the terms "idiopathic," " post-encephalitic," etc., are used to distinguish the varieties.

It is easy to diagnose a fully developed case of Parkinson's syndrome of any variety. Often there is an immediate impression of the condition which later proves to be correct, although at the time the impression was gained no adequate proof could be offered. However, it is sometimes difficult to diagnose cases that are just beginning to manifest themselves, or abortive cases, the so-called "formes frustes." The beginning and the course of Parkinson's syndrome of any variety is usually insidious and slow, and this makes the diagnosis particularly difficult.

In post-encephalitic Parkinson's syndrome it may be impossible to obtain a clear history of encephalitis, or the encephalitis may have occurred as long as 15 or 20 years previously, rendering some histories unreliable. The long so-called pseudo-neurasthenic stage of postencephalitic Parkinson's syndrome, with its manifold and vague symptoms, particularly contributes to the diagnostic confusion. Many and varied morbid conditions must be considered in the differential diagnosis in such cases: diverse degenerative and non-degenerative diseases of the brain, multiple sclerosis, exophthalmic goitre, senile tremor, familial tremor, arthritis, as well as psychoneurotic, neurasthenic, and hysteric states and various intoxications.

How difficult to some clinicians the diagnosis may be is drastically demonstrated by the fact that I have seen patients with unmistakable Parkinson's syndrome who previously had been subjected to encephalography and angiography in the search for a tumour.

\section{Tests in Early Diagnosis of Parkinson's Syndrome}

The early diagnosis of this condition has long engrossed investigators, and many tests have been devised for this purpose. Some of these are: (1) Abnormal position of hand and fingers, particularly of the thumb. (2) Easy exhaustibility of fine finger movements. (3) Positive push test: on a sudden push backward, the patient, standing erect, does not involuntarily dorsiflex the foot and toes on the affected side. (4) Arm-deviation test: When the patient, with his eyes closed, stands with arms outstretched in front of him there is a tendency for the arms to move inward and downward. (5) Overestimation of weight of objects placed in the affected hand. (6) Cogwheel rigidity of muscles: On quick passive stretching, the examiner feels short jerks in the stretched muscles of the patient. (7) Fixation of position test (a so-called postural reflex): The examiner places his thumb on the biceps tendon, and with the other hand passively flexes the forearm of the patient ; on every movement the thumb feels that the biceps tendon tightens so as to fixate the forearm in eyery given position. (8) Paresis of convergence of the eye muscles. (9) Hypometria, micro- graphia. All these tests have a sound physiological basis, and are useful. However, it cannot be said that they become manifest early in the disease.

For many years I hav been accustomed to base the diagnosis of early Parkinson's syndrome on the following tests: (1) Exaggeration of the orbicularis oculi reflex : closure of the eyes on tapping the glabella (Wartenberg, 1945). (2) Diminution of pendulousness of the arms in walking, and diminution of pendulousness of the legs. For the latter test the patient sits on the table with his legs hanging freely. The examiner lifts the legs, lets them swing freely, and appraises their swinging time. This time is conspicuously shorter in Parkinson's syndrome, particularly when only one side is affected (Wartenberg, 1951). (3) Increased resistance in the neck muscles as felt by the examiner when he moves the head of the patient in different directions.

This third test, based on the rigidity of the neck muscles, has seemed particularly useful, though the method is somewhat crude. However, its physiological rationale is sound.

\section{Tremor and Rigidity}

The fundamental motor disturbances in Parkinson's syndrome can be traced to two main factors-tremor and rigidity. Which of these is the more important? The first observers stressed tremor. This is understandable, since, both for the patient and for the doctor, it is the more conspicuous, the more striking manifestation of the disease, especially so since the tremor affects predominantly the distal parts of the extremities, and is more obvious and disturbing to the patient. I have seen many such patients with both tremor and rigidity who complained only of the tremor.

Later investigators stressed rigidity at the expense of tremor. The tendency has now become to regard tremor as the non-essential, secondary feature, and to emphasize the existence of paralysis agitans sine agitatione. "I for my part," said Lewy (1932), "reject paralysis agitans sine rigiditate." This is in accordance with my own experience. I have seen patients with Parkinson's syndrome of any variety showing rigidity without tremor. For me the more significant element of the pallidal syndrome is rigidity, which is always present. Wilson (1940, p. 799), however, said: "Examples of paralysis agitans sine rigiditate are seen once in a while; they include akinesis, tremor, sometimes Parkinsonian postures, but not hypertonia." Though there is certainly Parkinson's syndrome without tremor, I doubt very much that there is Parkinson's syndrome without rigidity. In cases in which allegedly there was no rigidity the technique of examining for tonus has probably been faulty.

What comes first in the syndrome, tremor or rigidity ? Many textbooks assert that in most cases tremor is the first sign. But it is admitted that occasionally tremor may appear some years after rigidity. Gowers (1893) found that rigidity may constitute " the first symptom of the disease." According to Wilson (1940, p. 791), "slowness and rigidity precede visible shaking in a considerable number." It is the post-encephalitic condition, particularly, that may show marked rigidity in the early stages, and only later may the tremor become apparent.

Where does rigidity in Parkinson's syndrome start? It is generally assumed that it starts in the proximal muscles of the limbs, particularly the upper. It is my belief that the rigidity starts in the neck and shoulder muscles, particularly in the flexors of the head. This leads to the sustained flexor position of the head, an early sign. As the disease advances and spreads this rigidity still remains more pronounced in the flexors of the head than in any other muscles of the body.

The textbooks point out the occurrence of the hemiparkinson syndrome. I would also like to stress the occurrence of a cervico-brachial type. This type is seen particularly in the idiopathic variety. In such cases the rigidity affects the muscles of the neck and shoulders predominantly or exclusively. The leg muscles are much less affected, or not affected at all. 


\section{Neck Rigidity}

My long-held view that rigidity in Parkinson's syndrome affects the muscles of the neck first and foremost I found also to be held by Vujic (1927). He speaks of "pronounced extrapyramidal hypertonia of the neck muscles," in cases of post-encephalitic Parkinson's syndrome. "The same hypertonia," says Vujic, " is often found in the limbs in less pronounced degree, sometimes much more distinctly in the arms than in the legs, and especially in the proximal parts.... Hypertonia is ascertained by means of passive movement of the head and limbs."

In photographs of some cases the prominence and tension of the sternocleidomastoid muscles which produce the forward bending of the head are conspicuous. "In the recumbent posture sternomastoids do not slacken " (Wilson, 1940, p. 794). How strongly this position of forward bending of the head is can be demonstrated convincingly by the following. When a patient with advanced Parkinson's syndrome is asked to lie supine, his occiput does not touch the supporting surface on which he lies. He keeps his head up from the surface indefinitely, even if he is admonished to relax (see illustration).

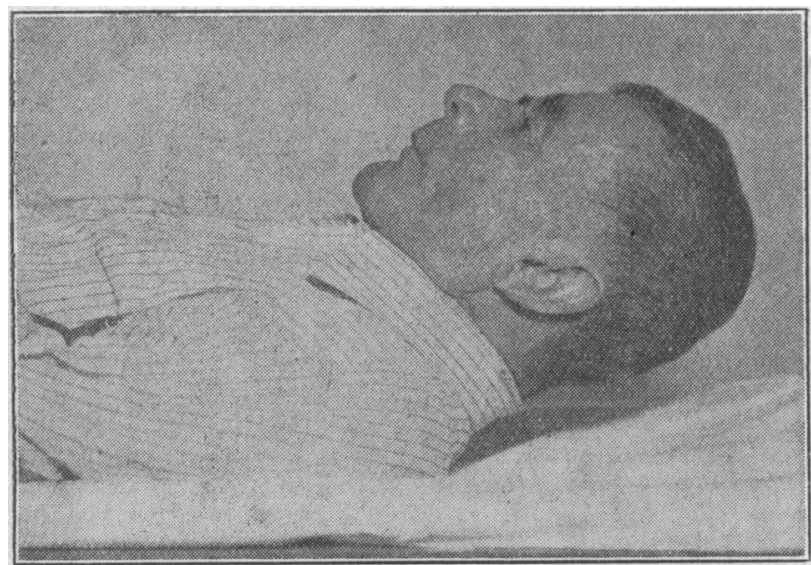

Advanced Parkinson's syndrome. Typical posture of head of patient lying supine: the head is kept flexed indefinitely and off the surface.

Professor Robert Bing has drawn my attention to the fact that Steck $(1926,1927)$ apparently was the first to describe this oreiller psychique (psychic pillow), which was seen in his patients with psychosis accompanied by extrapyramidal syndromes. Steck graphically illustrated this "psychic pillow." While the phenomenon was found by him in psychotic patients who had extrapyramidal signs, none of my patients was in any way psychotic, and the "psychic pillow" was due here exclusively to the extrapyramidal rigidity of the neck muscles. The term "psychic pillow" certainly cannot be applied to cases of Parkinson's syndrome. The "pillow" here is not "psychic" but grossly "organic." It is hardly correct to call it "psychic" even in Steck's cases with psychosis, since these show extrapyramidal syndromes to which this position of the head must be attributed in the first place.

From a physiological standpoint this early bending position of the head in Parkinson's syndrome is easy to understand. The postural factor is considered to be determined by the "loss of righting reflex" (Brock and Wechsler, 1927), fundamental for this disease. In Parkinson's syndrome there is a "dissolution of erectness ": (Kraus, 1924), a disintegration of the erect posture in man. The forward bending of the head is the very first expressir 7 of this disintegration.

If this be so, if indeed rigidity of the neck muscles is an early sign of Parkinson's syndrome, then the best test for this rigidity would be a test which demonstrates the rigidity of the flexors of the head. This, in fact, is the basis for the head-dropping test. .

\section{Technique of the Head-dropping Test}

The patient lies supine on a horizontal examining table, without a pillow. The surface should not be hard, but padded. $\mathrm{He}$ is made to relax completely and should not try to raise his head, as he is always inclined to do. He is asked to close his eyes, his attention is diverted, and he is engaged in conversation. His head rests on the table surface. The examiner places his left hand under the patient's occiput, the dorsum resting on the table and the occiput lying in the examiner's palm. Then, with his right hand, the examiner suddenly, unexpectedly, and briskly lifts the patient's head and allows it to drop.

In normal persons the head drops back on to the left hand of the examiner with force, like a dead weight. It is as if there were no tonus of the neck muscles at all, as if the head were attached to the body by a string. This complete lack of tonus is in gross contradistinction to the marked tonus the neck muscles exhibit when the patient is erect. One is amazed at the sharp impact with which the head falls heavily and inertly backwards, an impact felt distinctly by the examiner. The fall is so heavy that, were it to occur on a hard surface, the patient could sustain an injury. It even might hurt him if his head should happen to fall on a ring the examiner was wearing. It is essential that the patient be completely relaxed. In the rare instances where complete relaxation cannot be achieved the test must be given up for the time being. In any case it is advisable to repeat the test. If after several examinations varying results are obtained the heaviest drop counts; in all others it must be assumed that some active interference has taken place on the part of the patient.

The test can also be performed on the patient lying prone, or on either side, but it is much more easily performed and its results are less difficult to evaluate when the patient lies supine. The rigidity of the neck muscles is evidently greater in the flexors than in the retractors of the head. It must be assumed, therefore, that the dropping of the head is slower when the patient lies supine than when he lies prone. However, this is difficult to prove.

The most striking change from the normal in this headdropping test is seen in Parkinson's syndrome even in the earliest stages. Here the head drops stowly and gently; the downward movement is, so to speak, reluctant, hesitant, and lazy. The slow dropping of the head shows that, though the rigidity of the muscles in Parkinson's syndrome is generally diminished when the patient assumes a recumbent position, the rigidity of the neck muscles still remains considerable. In character this movement is the same as that felt by the examiner when he passively stretches a rigid extremity. The downward movement of the head maintains an even flow, is smooth, and is uniform from beginning to end. There is no sudden "give," no erratic unevenness. The smoothness and evenness of the movement are clearly seen in slow-motion pictures. If the patient interferes voluntarily and makes a movement with his head. this immediately can be distinguished as such.

The examiner is able to appraise the character of the motion not only by sight but by touch as well. In the palm of his hand he can feel the degree of impact of the dropping head. Compared with the violent impact in normal subjects. in Parkinson's syndrome the head drops softly and smoothly. The difference between the normal and the pathological is unmistakable, even in the earliest stages of the syndrome. I have seen quite a number of patients with Parkinson's syndrome who did not complain of rigidity anywhere, yet in whom the head-dropping test proved to be typical for this disease. In advanced cases the test is not necessary. Here the rigidity of the neck muscles is too obvious to need any special tests. As shown above, this rigidity is strikingly illustrated by the fact that the patient with advanced Parkinson's syndrome lying supine keeps his head bent and off the surface (see illustration). This is, so to speak, a "frozen" head-dropping test. In Parkinson's syndrome the test has 
been found abnormal so regularly, and this abnormality has been so clear and unequivocal, that 1 dare state that if the head-dropping test is normal the syndrome can be excluded.

\section{The Test in Differential Diagnosis}

The test is of great importance in differential diagnosis: Parkinson's syndrome versus senile tremor.

I have seen cases of tremor diagnosed by authorities as Parkinson's syndrome in which the head-dropping test was later found to be normal. This led to further clinical investigation which proved beyond doubt that the patient suffered from senile tremor and not Parkinson's syndrome. Again and again I have found that the latter diagnosis is made too often. The differential diagnosis between these two conditions may be very difficult, since they have many clinical features in common. It is true that there is a difference between senile and parkinsonian tremor, yet tremor per se gives no decisive clue for differential diagnosis. The outstanding characteristic of senile tremor-involvement of the head-may occur also in Parkinson's syndrome. It is therefore understandable that many authors, even quite recently, have maintained that the senile tremor is just a "forme fruste" of paralysis agitans. However, a differential diagnosis is of importance particularly with regard to prognosis, since senile tremor is a benign, non-progressive lesion, while Parkinson's syndrome is not. In this differential diagnosis, parkinsonian tremor versus senile tremor, the head-dropping test is of decisive importance.

The outstanding sign of the senile tremor is the invariable absence of rigidity. The head-dropping test, being the most sensitive test for rigidity, decides the issue. It is always unmistakably and completely normal in senile tremor.

Dr. Charles Kunkle tried this test on a 70-year-old woman who had had a typical essential heredofamilial tremor for 30 years. In a personal communication he states: "When put to the test her head dropped in entirely, normal fashion, like a true dead weight."

\section{Other Tests}

Next to the head-dropping test, the following have proved to be helpful in the early diagnosis of Parkinson's syndrome:

(a) Shoulder-shaking Test.-The examiner places his hands on the patient's shoulders, shakes them briskly by alternating movements to and fro, and observes the passive swinging of the arms thus produced. On the side affected by Parkinson's syndrome the range of the swinging is less than on the healthy side. When both sides are affected the diminution of swinging runs parallel to the rigidity. However, quite the opposite prevails in a cerebellar affection. Here the range of the swinging is greater on the affected than on the healthy side.

(b) Arm-dropping Test.-The examiner, standing in front of the patient, puts his hands on the sides of the patient's trunk, dorsum outward, at the level of the wrists. The patient is made to relax. With a sudden, quick, outward movement the examiner thrusts the patient's arms up and allows them to fall and strike the dorsum of his own hand. The examiner sees, feels, and hears that the impact of the falling arm on the side affected with Parkinson's syndrome is slowed up and not as heavy as that on the healthy side. The more the side is affected the softer and more retarded is the impact of the falling arm.

\section{(c) Pendulousness of the legs (as described above).}

A tremor may show the greatest similarity to Parkinson's syndrome, but when the head-dropping test and these three tests are normal then the syndrome can certainly be excluded. Thus far I have not seen any case of Parkinson's syndrome of any degree, of any variety, in which the head-dropping test was normal.

\section{Head-dropping Test in Extrapyramidal Diseases}

Since the neck muscles are a place of predilection in many other affections of the extrapyramidal system, it is understandable that an abnormal head-dropping test is found in such extrapyramidal conditions as Wilson's disease, torsion dystonia, striatal torticollis, and pallidal degeneration. Thus the dropping of the head as seen in Parkinson's syndrome indicates involvement of the neck muscles in extrapyramidal affections and even the degree of this involvement. It has proved helpful in cases of cortico-striato-spinal degeneration belonging to the group of spastic pseudosclerosis, and of olivo-ponto-cerebellar atrophy, pointing to the existing extrapyramidal component in these diseases. Generally speaking, a pathological head-dropping test in any complicated morbid condition indicates concomitant involvement of the extrapyramidal system.

We have thus far learned one outstanding characteristic of the head-dropping test: it shows striking changes in affections of the extrapyramidal system. There is another feature of this test which makes it valuable for differential diagnosis of extrapyramidal and pyramidal affections; it is that the head-dropping test does not show any marked or substantial change in pyramidal affections, not even in those in which both pyramidal tracts are affected intracranially.

A completely or nearly completely normal head-dropping test has been observed in patients with bilateral pyramidal lesions, which include an involvement of the pyramidal cortico-cervical tracts. These were cases of spastic tetraplegia with positive snout reflex, positive head retraction reflex, and markedly exaggerated jaw reflex. Some cases showed marked supranuclear bulbar ("pseudobulbar") signs and pathological laughing and crying. The common hemiplegia, of course, also shows a normal head-dropping test, and so does any affection of the cerebellum.

This being so, a pathological head-dropping test in diffuse affections of the brain points to extrapyramidal involvement. In diffuse hypertensive encephalopathy and in multiple sclerosis there may be such an extrapyramidal component. This can be ascertained and appraised by means of the headdropping test.

The statement just made, that affections of the pyramidal tracts do not influence the head-dropping test, must be modified somewhat by saying that they do not essentially affect the head-dropping test. Though it is true that cases of marked pyramidal involvement have been seen in which this test was normal, occasionally some modification of it has been seen in pyramidal lesions. But here the downward movement of the head never assumes the same character as that seen in Parkinson's syndrome. It is only somewhat uneven, jerky. However, the problem of the head-dropping test in pyramidal lesions requires further investigation. The minor deviation in the test as seen in pyramidal lesions is infinitely less evident than that seen in Parkinson's syndrome, in which it is so obvious as to be unmistakable. Therefore if in a brain lesion with pyramidal signs the head-dropping test is found as in Parkinson's syndrome, this points to concomitant involvement of the extrapyramidal nervous system.

To sum up: In the head-dropping test, the head of the patient lying supine is suddenly and briskly lifted and dropped by the examiner. A slow hesitant movement or a soft fall, a gentle hitting of the surface, is indicative of a lesion of the extrapyramidal system and constitutes a valuable diagnostic sign in Parkinson's syndrome.

\section{REFERENCES}

Brock, S., and Wechsler, I. (1927). Arch. Neurol. Psychiat.. Chicago, 17, 12. Gowers, W. R. (1893). A Manual of Diseases of the Nervous System. 2nd ed., 2, 646. Churchill. London.

Kraus, W. M. (1924). Ann. Med., 15, 67

Lewy, F. H. (1923). Die Lehre vom Tonus und der Bewegung, 9. Springer, Berlin.

Steck, H. (1926). Schweiz. Arch. Neurol. Psychlat., 19, 195.

Vuil (1927). Ibid., 20, 92.

Vujic, V. (1949). Acta med. Iugoslav.. 3, 133

artenberg. R. (1945). The Examination of Reflexes. D. 30 Y Year Book Publishers, Chicago.

Wibon, S. A. K. (1940). Neurology, 2, 791, 794. 799. Aroold Landon. 